\title{
Prediction of falls using a risk assessment tool in the acute care setting
}

\author{
Alexandra Papaioannou*1, William Parkinson ${ }^{2}$, Richard Cook ${ }^{3}$, \\ Nicole Ferko ${ }^{4,5}$, Esther Coker ${ }^{6}$ and Jonathan D Adachi ${ }^{1}$
}

\begin{abstract}
Address: ${ }^{1}$ Department of Medicine, McMaster University, Hamilton, Ontario, Canada, ${ }^{2}$ School of Rehabilitation Sciences, McMaster University, Hamilton, Ontario, Canada, ${ }^{3}$ Department of Statistics \& Actuarial Sciences, University of Waterloo, Waterloo, Ontario, Canada, ${ }^{4}$ Department of Clinical Health Sciences, McMaster University, Hamilton, Ontario, Canada, ${ }^{5}$ Innovus Research Inc., Burlington, Ontario, Canada and ${ }^{6}$ Hamilton Health Sciences, Hamilton, Ontario, Canada

Email: Alexandra Papaioannou* - papaioannou@hhsc.ca; William Parkinson -wparkinson@primus.ca; Richard Cook - rjcook@uwaterloo.ca; Nicole Ferko - nferko@innovus.com; Esther Coker - coker@hhsc.ca; Jonathan D Adachi - jd.adachi@sympatico.ca

* Corresponding author
\end{abstract}

Published: 21 January 2004

BMC Medicine 2004, 2:I
Received: 13 August 2003

Accepted: 21 January 2004

This article is available from: http://www.biomedcentral.com/I74|-70I5/2/I

(C) 2004 Papaioannou et al; licensee BioMed Central Ltd. This is an Open Access article: verbatim copying and redistribution of this article are permitted in all media for any purpose, provided this notice is preserved along with the article's original URL.

\begin{abstract}
Background: The British STRATIFY tool was previously developed to predict falls in hospital. Although the tool has several strengths, certain limitations exist which may not allow generalizability to a Canadian setting. Thus, we tested the STRATIFY tool with some modification and re-weighting of items in Canadian hospitals.
\end{abstract}

Methods: This was a prospective validation cohort study in four acute care medical units of two teaching hospitals in Hamilton, Ontario. In total, 620 patients over the age of 65 years admitted during a 6-month period. Five patient characteristics found to be risk factors for falls in the British STRATIFY study were tested for predictive validity. The characteristics included history of falls, mental impairment, visual impairment, toileting, and dependency in transfers and mobility. Multivariate logistic regression was used to obtain optimal weights for the construction of a risk score. A receiver-operating characteristic curve was generated to show sensitivities and specificities for predicting falls based on different threshold scores for considering patients at high risk.

Results: Inter-rater reliability for the weighted risk score indicated very good agreement (interclass correlation coefficient $=0.78$ ). History of falls, mental impairment, toileting difficulties, and dependency in transfer / mobility significantly predicted fallers. In the multivariate model, mental status was a significant predictor $(P<0.001)$ while history of falls and transfer / mobility difficulties approached significance $(P=0.089$ and $P=0.077$ respectively). The logistic regression model led to weights for a risk score on a 30 -point scale. A risk score of 9 or more gave a sensitivity of $91 \%$ and specificity of $60 \%$ for predicting who would fall.

Conclusion: Good predictive validity for identifying fallers was achieved in a Canadian setting using a simple-to-obtain risk score that can easily be incorporated into practice. 


\section{Background}

Falls account for at least $40 \%$ of all accidents in hospital [1]. Risk of hip fracture was found to be 11 times greater in hospital patients compared to those in the community [2]. Patient characteristics implicated in falls that occur in hospitals include history of falls, difficulty in transfers or ambulating, dizziness and balance, delirium, visual impairment, medications, incontinence and toileting frequency [3-5].

Clinical prediction rules are tools designed to predict health outcomes and assist health care professionals plan patient care. These tools typically include three or more risk factors from a patient's history or physical exam that predict an outcome such as falls [6]. The STRATIFY tool was developed and validated in the United Kingdom to predict falls occurring in hospital. The tool contains five clinical factors associated with falling (e.g. previous falls, mental impairment) with a simple scoring system. The tool incorporates many of the features that instrument developers desire including 1) predictive validity: high sensitivity and specificity for predicting falls, 2) feasibility; the items are easily and rapidly assessed by nursing staff with minimal staff training required, and 3) reproducibility; the predictive variables and the decision rule were tested in different geriatric settings [7].

However the STRATIFY tool has limitations. Altman $[8,9]$ pointed out that falls, rather than patients, were outcomes in the STRATIFY study, which could inflate predictive validity. Specifically, of 217 patients, 71 falls occurred (approximately a 30\% rate of falls) [6]. Prediction might also have been weakened by the absence of item weighting as certain patient characteristics may have greater value in predicting falls. Price [10] emphasized that the use of data on falls in hospital to update risk scores in the STRATIFY study, would inflate the calculated predictive validity and decrease useful prediction. In fact, the majority of falls occur in the first week of hospitalization $[11,12]$. Finally, the use of items in the tool having varying interpretations (e.g. agitation) may compromise reproducibility. Ideally, each question in a clinical tool should be interpreted in the same way.

We tested the predictive validity of the STRATIFY variables in a Canadian setting, with the objective of determining if it predicts fallers rather than patient falls. We constructed a weighted risk score based on the components of the STRATIFY tool and examined its predictive validity.

\section{Methods}

\section{Setting and participants}

Data were collected over a 6-month period in 2000 at Hamilton Health Sciences, Hamilton, Ontario, Canada, a multi-site teaching hospital. Patients over 65 years of age admitted consecutively to four general medical units having a total of 114 beds were assessed. Palliative and critical care patients were excluded.

\section{Definition of clinical outcome}

The clinical outcome we studied was "fallers" versus "nonfallers". "Fallers" were those having one or more falls during hospitalization. A fall was defined as an individual involuntarily coming to rest on the ground or surface lower than their original station [13]. The attending nurse documented each fall on an incident report and in the patient chart. Nurses completing the risk assessment tool were blinded to the rationale for assessing predictor variables. Incident reports noted time of fall, location, injury sustained, type of fall, and potential causative factors. Only falls occurring after screening were included as an outcome.

\section{Predictive variables: identification and definition}

Five patient characteristics found to be risk factors for falls in the British STRATIFY study [7] were assessed. Items were modified for the Canadian health care system to include the definitions of the risk factor to potentially increase reproducibility (Table 1 [see Additional file 1]). Mental status was divided into three concepts, "disorientation", "confusion", and "agitation" and definitions were added. Similarly, "vision impairment" was assessed based on four questions and "history of falls" was divided into two questions. Toileting assessment remained one item as in STRATIFY but the wording was altered and a definition was added. As in the original tool the "transfer and mobility" measure was taken from the Barthel Index [14] and was the sum for transfer ( 0 to 3 ) and mobility ( 0 to 3 ) scores for a total ranging from of 0 (dependent) to 6 (independent).

\section{Screening assessment scoring}

The nurses completing the screening tool had a 10-min orientation session run by the clinical nurse specialists who were part of the investigative team. The assigned nurse collected screening variables 24 to 48 hours after the patient was admitted to hospital in a 5-min bedside session.

To calculate predictive validity, each of the five items were scored dichotomously as $1=$ present, and $0=$ absent for items 1 to 4 . The risk factors were deemed to be "present" if one or more of the statements were considered within each domain (Table 1 [see Additional file 1]). For example, mental impairment was present if one or more of confusion, disorientation or agitation were scored "yes". If none of these variables were present, then the risk factor was scored as "absent". Scores of 0 to 3 for the transfer and mobility sub-score corresponded to the presence of the 
Table I: Odds ratio (OR) and $95 \%$ confidence intervals $(\mathrm{Cl})$ from the univariate and multivariate logistic regression models.

\begin{tabular}{|c|c|c|c|c|c|c|}
\hline \multirow[t]{2}{*}{ Item } & \multicolumn{3}{|c|}{ Univariate Analysis } & \multicolumn{3}{|c|}{ Multivariate Analysis } \\
\hline & OR & $95 \% \mathrm{Cl}$ & P-value & OR & $95 \% \mathrm{Cl}$ & P-value \\
\hline History of falls & 2.47 & $(1.23,4.96)$ & 0.011 & 1.88 & $(0.91,3.89)$ & 0.089 \\
\hline Mental Status & 6.07 & $(2.86,12.86)$ & $<0.001$ & 4.06 & $(1.81,9.16)$ & $<0.001$ \\
\hline Vision Impaired & 1.02 & $(0.5 \mathrm{I}, 2.05)$ & 0.946 & 1.09 & $(0.53,2.24)$ & 0.824 \\
\hline Toilet Difficulty & 2.79 & $(1.37,5.67)$ & 0.005 & 1.20 & $(0.52,2.75)$ & 0.669 \\
\hline Transfer / Mobility & 3.71 & $(1.83,7.50)$ & $<0.001$ & 2.10 & $(0.92,4.78)$ & 0.077 \\
\hline
\end{tabular}

risk factor and scores of 4 to 6 were considered to represent absence of the risk factor.

\section{Statistical analyses}

Each of the five items was assessed individually and collectively for their predictive power based on logistic regression models. The variables were also used to produce an overall risk score using two approaches. First, an unweighted risk score (r) was computed by simply counting the number of risk factors present. This gave a risk score ranging from 0 to 5 , as in the original STRATIFY study.

Second, a weighted risk score was obtained based on the regression coefficients from the multivariate logistic regression model in which the outcome was the fall status of the patient. Specifically, the relative magnitude of the beta coefficients from the multivariate logistic model reflects the relative prognostic strength of the risk factors when they are jointly considered. Therefore the relevant information for the construction of the weights is the relative size of the beta coefficients and any weights, which preserve these ratios, will preserve good predictive validity (see Appendix [Additional File 1] for further details).

To consider predictive validity in terms of sensitivity and specificity one must consider two populations, "fallers" and "non-fallers". Two receiver operating characteristic curves (ROC) were constructed to display the varying specificity and sensitivity values applicable to the range of possible thresholds of unweighted $(0-5)$ and weighted risk $(0-30)$ scores that could be used to classify patients as high risk for falling or not. All analyses were performed using the Statistical Analysis System (SAS release 8.1).

\section{Inter-rater reliability}

Two nurses independently assessed a sub-sample of 35 patients. The order of assessments was random and assessors were blind to the findings of the other nurse. The required sample size was estimated at 33 patients, with power $=0.80$ and alpha $=0.05$ using published power tables [15]. The inter-class correlation coefficient (ICC) was computed for the weighted and un-weighted risk scores. The kappa statistic was computed on reliability of classification into "high" versus "normal" risk based on the optimal threshold score.

\section{Results}

Over 6 months, 620 patients were screened for falls. The mean age was 78 years (SD 7.7) and 338 (54.5\%) patients were female. The diagnoses most responsible for hospitalization in the sample were circulatory disorders (45.2\%), respiratory disorders $(20.8 \%)$, digestive disorders $(4.0 \%)$ and mental disorders $(2.9 \%)$. Diagnosis was not predictive of falls. Thirty-four patients (5.5\%) fell at least once during their hospitalization and there were a total of 77 falls. In total, 171 patients (27.6\%) had a history of falls at admission.

\section{Predictive variables}

Based on univariate logistic regression (Table 1 ) history of falls prior to admission $(\mathrm{P}=0.011)$, mental status (confused, disoriented or agitated $)(\mathrm{P}<0.001)$, toileting difficulties $(\mathrm{P}=0.005)$ and transfer $/$ mobility difficulties $(\mathrm{P}<$ $0.001)$ predicted falls. When the inter-correlations between these independent variables were controlled for using multiple logistic regression (Table 1), only mental status $(P<0.001)$ remained a significant predictor. In terms of the magnitude of the associations from this multivariate model the odds of falling increased over four fold in individuals with mental impairment (odds ratio (OR) $=4.06 ; 95 \%$ CI: 1.81,9.16). History of falls and transfer $/$ mobility difficulties incurred an approximate two-fold increase in risk while approaching significance $(P=0.089$ and $\mathrm{P}=0.077$ respectively).

When length of stay was added to the multivariate model, the odds ratios associated with each risk factor were slightly attenuated. However, the pattern of results remained the same and mental status was most predictive, followed by history of falls, and then transfer/mobility difficulties. 


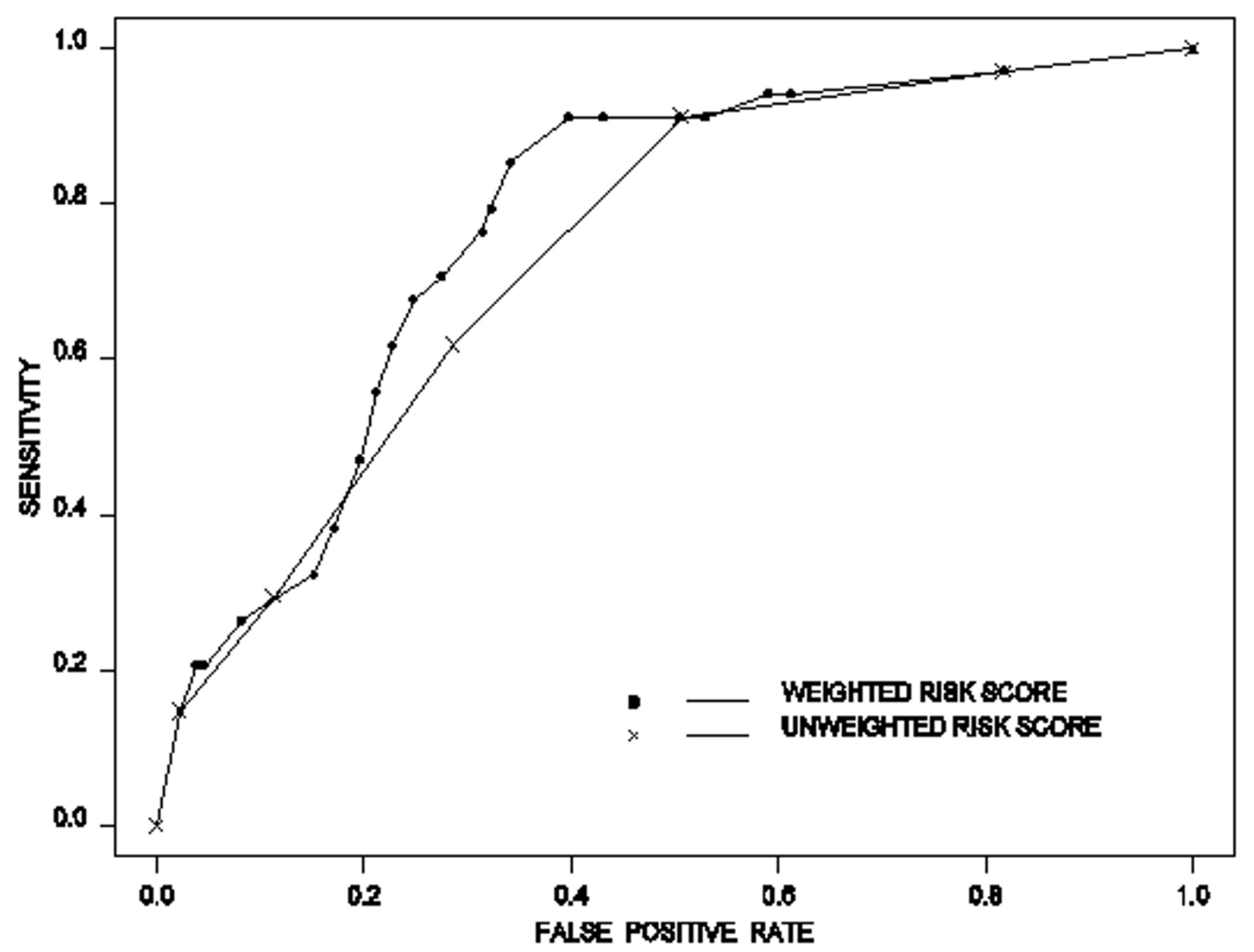

Figure I

Receiver operating characteristic curve $(\mathrm{ROC})$ for weighted $(0-30)$ and unweighted $(0-5)$ risk score

A weighted risk formula (Appendix I) was derived which shows the relative magnitude of the regression coefficients from the multivariate logistic regression model. This formula can easily be used to calculate the weighted risk score.

\section{ROC curves}

The ROC curves based on the unweighted and weighted risk scores are given in Figure 1 and sensitivities and specificities are displayed for a subset of possible threshold values in Table 2. As the weighted risk score $(\mathrm{R})$ is increased from 0 , the sensitivity falls very little until one reaches a false positive rate of about 0.40 , which corresponds to $R=9$. Balancing sensitivity and specificity, we selected this as the ideal cut-off score, which results in a sensitivity of $91.2 \%$ (95\% CI: 81.6, 100.7) and specificity of $60.2 \%$ (95\% CI: $56.3,64.2)$. With a threshold of 9 , the tool would correctly classify over $90 \%$ of those patients at high risk for falling and over $60 \%$ of those at normal risk. When the cut-off score increases to 20, specificity increases slightly (78.8\%), however, sensitivity significantly falls (55.9\%).

The ROC curve for the un-weighted risk score (r) (Figure 1) had poorer predictive validity. For example, when two or more risk factors are present $(r \leq 2)$, sensitivity was $91.2 \%$, but specificity was only $49.3 \%$. Increasing $r$ to 3 or more risk factors resulted in an increase in specificity to $71.3 \%$ but sensitivity dropped to an unacceptable $61.8 \%$. 
Table 2: Sensitivities and specificities for threshold of weighted risk score $(0-30)$

\begin{tabular}{ccccc}
\hline Risk Score & False Positive Rate & False Negative Rate & Sensitivity & Specificity \\
\hline 0 & 100.0 & 0.0 & $100.0(100.0,100.0)$ & $0.0(0.0,0.0)$ \\
1 & 81.7 & 2.9 & $97.1(91.4,102.7)$ & $18.3(15.1,21.4)$ \\
2 & 61.3 & 5.9 & $94.1(86.2,102.0)$ & $38.7(34.8,42.7)$ \\
3 & 59.0 & 5.9 & $94.1(86.2,102.0)$ & $41.0(37.0,44.9)$ \\
6 & 52.9 & 8.8 & $91.2(81.6,100.7)$ & $47.1(43.1,51.1)$ \\
7 & 50.5 & 8.8 & $91.2(81.6,100.7)$ & $49.5(45.4,53.5)$ \\
8 & 43.0 & 8.8 & $91.2(81.6,100.7)$ & $57.0(53.0,61.0)$ \\
9 & 39.8 & $\mathbf{8 . 8}$ & $\mathbf{9 1 . 2 ( 8 1 . 6 , 1 0 0 . 7 )}$ & $\mathbf{6 0 . 2 ( 5 6 . 3 , 6 4 . 2 )}$ \\
10 & 34.1 & 14.7 & $85.3(73.4,97.2)$ & $65.9(62.0,69.7)$ \\
13 & 32.3 & 20.6 & $79.4(65.8,93.0)$ & $67.7(64.0,71.5)$ \\
14 & 31.4 & 23.5 & $76.5(62.2,90.7)$ & $68.6(64.8,72.4)$ \\
15 & 27.5 & 29.4 & $70.6(55.3,85.9)$ & $72.5(68.9,76.1)$ \\
16 & 24.7 & 32.4 & $67.6(51.9,83.4)$ & $75.3(71.8,78.7)$ \\
17 & 22.7 & 38.2 & $51.8(45.4,78.1)$ & $77.3(73.9,80.7)$ \\
20 & 21.2 & 44.1 & $47.1(39.2,72.6)$ & $78.8(75.5,82.1)$ \\
21 & 19.6 & 52.9 & $38.2(21.9,54.6)$ & $80.4(77.2,83.6)$ \\
22 & 17.1 & $32.4(16.6,48.1)$ & $82.9(79.9,86.0)$ \\
23 & 15.2 & 67.6 & $84.8(81.9,87.7)$ \\
24 & 8.2 & 73.5 & $26.5(11.6,41.3)$ & $91.8(89.6,94.0)$ \\
27 & 4.6 & 79.4 & $20.6(7.0,34.2)$ & $95.4(93.7,97.1)$
\end{tabular}

\section{Inter-rater reliability}

The inter-rater reliability between the two nurses using the weighted risk score gave an ICC of 0.78 (95\% CI: 0.60, $0.88)$. The reliability of the binary classification of high / normal risk gave kappa $=0.63(95 \% \mathrm{CI}: 0.36,0.90)$. The unweighted risk score gave a similar reliability (ICC $=$ 0.68 ; 95\% CI: $0.46,0.83$ ).

\section{Discussion}

Falls in the elderly are often a symptom of acute medical problems in combination with underlying risks such as medications, postural hypotension and lower extremity weakness. Identifying those at risk allows targeted assessment and intervention such as a review of medications and environmental modifications [16]. This study demonstrated good predictive validity for the modified SRATIFY tool to identify individuals at risk of falling in acute care. With a risk score of 9 , sensitivity was $91 \%$ and specificity was $60 \%$. The falls risk assessment tool can be easily incorporated into practice without added burden to the patient. The findings were achieved with a conservative methodology in which the outcome measure was the patient (i.e. fallers), rather than falls, and the risk score was generated before any falls. Despite minimal nurse training and short completion time, we were able to obtain very good inter-rater reliability $($ ICC $=0.78)$. A recent analytic review of falls risk assessment tools found that only two of five tools used in acute care with a sensi- tivity over 80 described how long the tool took to complete and only one had findings reproduced by other investigators. Many did not report inter-rater reliability [17].

Risk factors included in screening for falls in hospitalized patients have largely been consistent across studies with varying methods. Findings have repeatedly emphasized falls history, mental impairment, toileting frequency, and general mobility as predictive variables for falls $[7,18-23]$. Nevertheless, it is not yet clear how to maximize prediction. The variables included in different studies do not overlap entirely and some studies incorporate variables with poor or inconsistent predictive validity. For example, visual impairment had poor predictive value in our study and no significant predictive value in Morse's study [24]. Oliver et al. included visual impairment as a variable in STRATIFY based on an initial study phase in which it was moderately predictive $(\mathrm{OR}=3.55)$, and appeared to rank fifth strongest among 10 clinical variables described [7]. However, their design did not include a control for intercorrelations among risk factors. Also, relevant to optimizing prediction is the fact that Morse's study [24] and ours are the only ones to include weightings derived from quantitative analysis. Item weighting was clearly important to optimize prediction. 
Studies have also differed in the suggested ideal risk score cut-offs to consider patients in the "at risk" group. Whether our suggested cut-off of 9 is ideal for different hospital settings is not known. One approach is to use the cut-off that maximizes predictive power mathematically. Practitioners in different settings may adjust the trade-off between sensitivity and specificity, based on differing falls rates, values, laws, funding and other factors.

Our finding of poor predictive validity with the unweighted items does not clearly amount to poor generalization across settings because the items and protocol were changed. Studies involving tests of prediction tools in new locations have found results that are weaker than the original findings [25]. The difficulty obtaining generalized (i.e. reproducible) effects is concerning. One explanation may be that the base rate for occurrence of a clinical outcome is known to affect positive predictive value $[24,25]$. Our base rate for falls was $5.5 \%$, which is lower than that found in the British study and may have contributed to lower predictability. Another possibility is that prediction may only be consistent among patients with similar characteristics, resulting in generalization across some settings and not others.

A potential methodological limitation is uncertainty about patient incident reports, which may not capture all falls, however our documented rate of falls was similar to previous years in our setting. There is also the possibility that completing risk assessments influences how nurses respond to patients in terms of falls prevention strategies (i.e. Hawthorne effect). It is unknown if this factor affected the true rate of falls in our setting. However, it is predicted that this effect is likely minimal given that strong consistent falls prevention strategies were not in place at the time of the study. Another potential limitation is that despite some changes to scale items taken from STRATIFY to improve reproducibility; there is still room for error. For example, patient recall of falls may not be accurate and consistent. This may have accounted for an inter-rater reliability that was lower than ideal. The problem stresses a potential need to improve operational definitions of risk variables to ensure reproducibility in measuring items such as mental status. It may help to have consensus among investigators on key issues including: which variables to include, operational definitions of risk constructs, the duration over which risk is assessed (i.e. within 24 or 48 hours), the way in which users should be trained, and what the appropriate outcomes are (e.g. falls versus fallers). Further replication of our study in other settings will help to correct upon these limitations and improve generalizability.

\section{Conclusions}

We found good predictive validity for identifying fallers in hospital in a Canadian setting after weighting risk factors. Assessment of fall risk is one of the essential quality indicators for care of the older adult.

\section{Competing Interests}

None declared.

\section{Authors' contributions}

$\mathrm{AP}, \mathrm{WP}, \mathrm{JA}$ and EC were involved with study design. AP, WP, EC and NF were involved with study development, co-ordination and data collection. $\mathrm{AP}, \mathrm{NF}$ and $\mathrm{RC}$ were involved with data analysis. All authors were involved in preparing and writing the manuscript and approved the final version.

\section{Additional material}

\section{Additional File 1}

Appendix I: Falls Risk Screening Tool and Sample Calculations. Appendix I: Falls Risk Screening Tool and Sample Calculations. A description of how the weighted risk score formula was derived is described in this file. The falls risk screening tool is provided, with a patient example demonstrating how to calculate the overall risk score.

Click here for file

[http://www.biomedcentral.com/content/supplementary/17417015-2-1-S1.doc]

\section{Acknowledgements}

We wish to thank Tish Butson and Cathy Reiss from the Hamilton Health Sciences for their assistance in study nurse orientation and co-ordination of the study. This study was supported by a grant through the Hamilton Health Sciences Foundation. Dr. A Papaioannou is an Ontario Ministry of Health Career Scientist. Dr. RJ Cook is an investigator for the Canadian Institutes for Health Research.

\section{References}

I. Groves JE, Lavori PW, Rosenbaum JF: Accidental injuries of hospitalized patients. A prospective cohort study. Int J Technol Assess Health Care 1993, 9: 139-44.

2. Uden G, Nilsson B: Hip fracture frequent in hospital. Acta Orthop Scan 1986, 57:428-430.

3. Hendrich A, Nyhuis A, Kippenbrock T, Soja ME: Hospital falls: Development of a predictive model for clinical practice. Applied Nursing Research 1995, 8(3): 129-139.

4. Stevenson B, Mills EM, Welin L, Beal KG: Falls risk factors in an acute-care setting: a retrospective study. Can J Nurs Res 1998, 30(I):97-III.

5. Chu LW, Pei CK, Chiu A et al.: Risk factors for falls in hospitalized older medical patients. J Gerontol A Biol Sci Med Sci 1999, 54:M38-M43.

6. Laupacis A, Sekar N, Stiell IG: Clinical prediction rules: a review and suggested modifications of methodological standards. JAMA 1997, 277:488-494.

7. Oliver D, Britton M, Seed P, Martin FC, Hopper AH: A 6-point risk score predicted which elderly patients would fall in hospital. BMJ 1998, 3 I 5:1049-1053.

8. Altman DG: Study to predict which elderly patients will fall shows difficulties in deriving and validating a model. BMJ I997, 315:1309. 
9. Altman DG, Bland JM: Statistics Notes: Units of analysis. $B M J$ 1997, 3 I 4: 1874

10. Price C, Suddes M, Oshea D: Predicting ward falls. Validation of a quick nurse-led assessment of falls risk. Age and Aging 1998, 27(Suppl I):22.

11. Uden G, Nilsson B: Hip fracture frequent in hospital. Acta Orthop Scan 1986, 57:428-30.

12. Uden G: Inpatient accidents in hospitals. J Am Geriatr Soc 1985, 33:833-4I.

13. Tideiksaar R: Falling in old age: Prevention and management 2 nd edition. New York, NY: Springer; 1997.

14. Collin C, Wade DT, Davies S, Horne V: The Barthel ADL index: a reliability study. Int Disability Stud 1988, 10:61-69.

15. Donner A, Eliasziw M: Sample size requirements for reliability studies. Stat Med 1987, 6(4):44I-448.

16. Rubenstein LZ, Powers CM, MacLean CH: Quality indicators for the management and prevention of falls and mobility problems in vulnerable elders. Ann Intern Med 200I, 135:686-693.

17. Perell KL, Nelson A, Goldman RL et al.: Fall risk assessment measures: an analytic review. J Gerontol A Biol Sci Med 200I, 56(I2):M76I-766.

18. Mosley A, Calindo-Ciocon D, Peak N, Jo West M: Initiation and Evaluation of a Research-based fall prevention program. J Nurs Care Qual 1998, I3(2):38-44.

19. Spellbring AM, Gannon ME, Kleckner T, Conway K: Improving safety for hospitalized elderly. J Gerontological Nursing 1988, I4(2):31-37.

20. Heslin K, Towers J, Leckie $C$ et al:: Managing falls: Identifying population-specific risk factors and prevention strategies. In: Key Aspects of Elder Care Edited by: Funk SG, Tornquist EM. New York, Springer Publishing Company; 1997:17-37.

21. Raiche M, Hebert R, Prince F, Corriveau H: Screening older adults at risk of falling with the Tinetti balance scale. Lancet 2000 , 356: 1001 .

22. Schmid N et al.: Reducing patient falls: A research-based comprehensive fall prevention program. Military Medicine 1990, 155:202-209.

23. McCollam E: Evaluation and implementation of a researchbased falls assessment innovation. Nursing Clinics of North America 1995, 30(3):507-5|4.

24. Morse JM, Black C, Oberle K, Donahue P: A prospective study to identify the fall prone patient. Soc Sci Med 1989, 28(I):8I-86.

25. Coker E, Oliver D: Evaluation of the STRATIFY falls prediction tool on a geriatric unit. Outcomes Manag 2003, 7(I):8-14.

\section{Pre-publication history}

The pre-publication history for this paper can be accessed here:

http://www.biomedcentral.com/1741-7015/2/1/prepub

Publish with Biomed Central and every scientist can read your work free of charge

"BioMed Central will be the most significant development for disseminating the results of biomedical research in our lifetime. "

Sir Paul Nurse, Cancer Research UK

Your research papers will be:

- available free of charge to the entire biomedical community

- peer reviewed and published immediately upon acceptance

- cited in PubMed and archived on PubMed Central

- yours - you keep the copyright
BiolMedcentral 